\title{
COMPORTAMENTO DELLE ONDE DI RAYLEIGH IN UN MEZZO FIRMO-ELASTICO INDEFINITO
}

\author{
Pietro Cili oi
}

Supponiamo rhe il piano xy di una terna di assi rateriani (o) situicea la superficie libera del mezzo: l'ase $\approx$ sia rivolo verso l’interno del mezzo stesso.

Le equazioni generali dei piecoli moli in un corpo firmo-claclieo isotropo e omogeneo sono !e seguenli:

$$
\begin{aligned}
& 0 \frac{\partial^{2} u}{\partial l^{2}}=\left\{(i+u)+\left(i^{\prime}+u^{\prime}\right) \frac{\partial}{\partial t}\right\} \cdot \frac{\partial i}{\partial x}+\left(u+u^{\prime} \frac{\partial}{\partial t}\right) u_{u} u \\
& \varrho \frac{\partial^{2} v}{\partial l^{2}}=\left\{(\lambda+u)+\left(\lambda^{\prime}+\mu^{\prime}\right) \frac{\partial}{\partial l}\right\} \cdot \frac{\partial \eta}{\partial y}+\left(\mu+\mu^{\prime} \frac{\partial}{\partial l}\right) u_{. z} v
\end{aligned}
$$

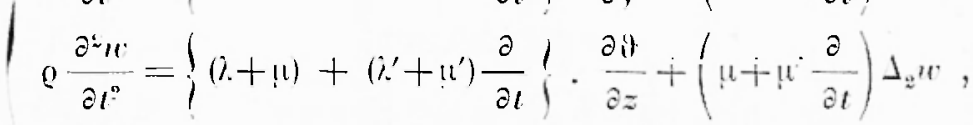

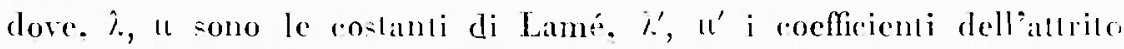
interno di rolume ed equiroluminale $c$ il esprime la diatiazione:

$$
i=\frac{\partial u}{\partial x}+\frac{\partial v}{\partial y}+\frac{\partial u}{d z} \text {. }
$$

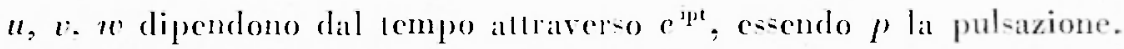
Pertinto

$$
\left\{\begin{array}{l}
{\left[i+u+i p\left(i^{\prime}+u^{\prime}\right)\right] \frac{\partial u}{\partial x}+\left(u+i p u^{\prime}\right) \Delta_{v} u+0 p^{z} u=0} \\
{\left[i+u+i p\left(\dot{r}^{\prime}+u^{\prime}\right)\right] \frac{\partial i}{\partial y}+\left(u+i p u^{\prime}\right) \Delta_{z} v+0 p^{2} v=0} \\
{\left[i+u+i p\left(i^{\prime}+1-u^{\prime}\right)\right] \frac{\partial i}{\partial z}+\left(u+i p u^{\prime}\right) \Delta_{1} u+e p^{2} u=0 .}
\end{array}\right.
$$

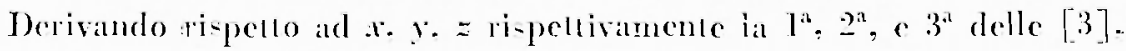
c commando nembro a membro, it ha

$$
\left(\Delta_{2}+h^{2}\right) i=0
$$


essendo

$$
h=\frac{e \mu^{2}}{i+2 u+i p\left(\mu^{\prime}+2 u^{\prime}\right)}
$$

Dividendo le $[3]$ per $u+i p \iota^{\prime} \leqslant \mathrm{i}$ lı

$$
\frac{i+u+i p\left(\hat{\kappa}^{\prime}+u^{\prime}\right)}{u+i p u^{\prime}} \cdot \frac{\partial i}{\partial z}-\bigsqcup_{2} i i-\frac{\partial p^{2}}{u+i p u^{\prime}} u=0
$$

Poniano

Alloria

$$
k^{2}=\frac{0 \mu^{2}}{\mu+i p \iota^{\prime}}
$$

e le [3] divengono

$$
\frac{\hat{h}+u+i p\left(i^{\prime}+u^{\prime}\right)}{!+i p !^{\prime}}=\frac{h^{z}}{h^{2}}-1
$$

$$
\left(\Delta_{2}+h^{2}\right) u=\left(1-\frac{h^{2}}{h^{2}}\right) \frac{\partial i}{\partial x} .
$$

En integrale particolare delle [7] i cestituito dalla soluzione

$$
\mu=-\frac{1}{h^{2}} \frac{\partial \hat{i}}{\partial x} \quad ; \quad v=-\frac{1}{h^{2}} \frac{\partial \hat{i}}{\partial y} ; u=-\frac{1}{h^{2}} \frac{\partial i}{\partial z} .
$$

Se a, $\beta, \gamma$ sono le componenti dello spostamento delle onde trasrersali. Sarà

e dalle $[7]$ conscg!t:

$$
\frac{\partial u}{\partial x}+\frac{\partial 3}{\partial y}+\frac{\partial y}{\partial z}=-0
$$

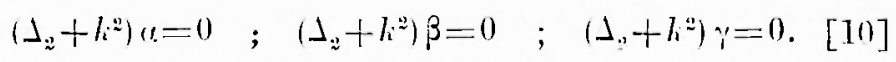

Le componenti delío spostamento dipendono da $x$. y mediante la funzione $e^{\mathrm{i}(\mathrm{x})} \cdot \cos f_{\mathrm{g}} g$ muncti complessi. Si ha

$$
\left.\frac{\partial^{2} i}{c^{2} x}==-j^{*}\right) \quad, \quad \frac{\partial^{2} i}{\partial y^{2}}=-g^{2} i \text {. }
$$

Lá [1] diviene

$$
\left(\frac{\partial}{\partial z^{2}}+h h^{2}-f^{2}-g^{2}\right) i=0
$$

$P_{0}=10$

si hat

$$
r^{2}=f^{2}+g^{2}-h^{2},
$$

$$
i=P e^{-r z}+Q e^{r z},
$$


Mat $r$ ècheralmente numero complesoo, $r=r^{\prime}-i r^{\prime \prime}$; con-eque

$$
i=P e^{-r / 2} e^{-i \mathrm{r} / z}+Q e^{\mathrm{r} / z} e^{\mathrm{s} r / z} \text {. }
$$

Vale solo il l" termine del $\underline{2}^{3}$ membro, ron esponente negativo. Pertanto $Q=0$. Prescindendo dal valore di $P$. espreso da $e^{i(p t+f x+g y)}$, potremo arivere

$$
\text { i) }=\epsilon^{-\mathbf{r} \mathbf{x}} \text {. }
$$

Ia soluzione particolate e-pres-a dalle 18$]$ diviene allora

$$
u=-\frac{i f}{h^{2}} \epsilon^{-r} ; v=-\frac{i g}{h^{2}} e^{-r z} ; u=\frac{r}{h^{2}} e^{-r} \text {. }
$$

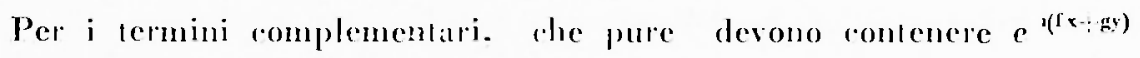
come litlori, le [10] divengono

$$
\left(\frac{\partial^{2}}{\partial x^{2}}+h^{2}-f^{2}-g^{3}\right) \mu=0, \text { ecc., }
$$

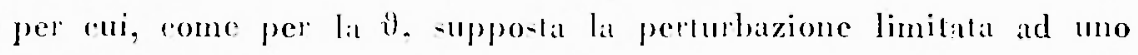
trato superficiale.

dove

$$
u=A e^{-s z} \quad ; \quad \beta=B e^{-s x} \quad ; \quad \gamma=C e^{-s x},
$$

$$
s^{*}=j^{*}+g^{*}-l^{2} \text {. }
$$

Poiche i coeffeienti nelle [16] romengono, guali funzioni di $x, y$

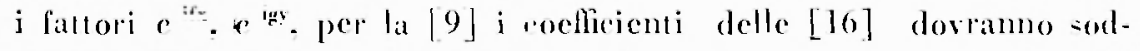
distiale allal relazione

$$
\text { if } 1+i g B-s C=0
$$

I valori completi di $u, r$,

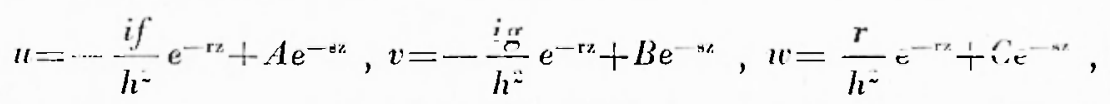

in rui A. B, C -ono sogretle alla [18].

Pas-iamo alle condizioni da soddisfare alla -uperfacie libera.

Per ze=0 devono ammuliar-i le due tensioni tangenziali

$$
\begin{aligned}
& T_{2}=\left(u+i P u^{\prime}\right)\left(\frac{\partial u}{\partial x}+\frac{\partial u}{\partial z}\right) \\
& T_{1}=(u+i p \mu)\left(\frac{\partial v}{\partial x}+\frac{\partial u}{\partial y}\right) .
\end{aligned}
$$


Ricordando che $u, v, w$ sono fumzioni di $x y$ attraverso wli esponenziali $e^{\text {ix }}, e^{\text {jgy }}$ si ha

$$
\frac{\partial v}{\partial x}=i g \frac{r}{h^{n}} e^{-\cdots}-B s e^{-\cdots} \quad ; \quad \frac{\partial w}{\partial y}=i g \frac{r}{h^{2}} e^{-\mathrm{r} 2}+i g C e^{--s 2},
$$

e analogamente per $\frac{\partial w}{\partial x}$ e $\frac{\partial u}{\partial z}$.

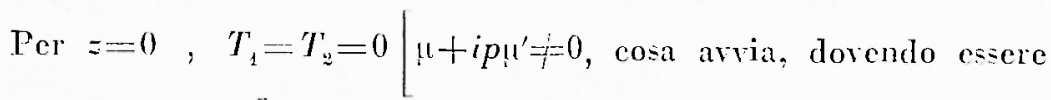
altrimenti $\left.u=u^{\prime}=0\right]$ ¿

$$
s B=\frac{2 i g r}{h^{2}}+i g C \quad, \quad s A=\frac{2 i f r}{h^{2}}+\imath l C .
$$

Sostituendo le [20] nelle $[18]$ si ha

cioe

$$
i f\left(\frac{2 i f r}{h^{2} s}+i f \frac{C}{s}\right)+i g\left(\frac{2 i g r}{h^{2} s}+\frac{i g C}{s}\right)-s C=0,
$$

$$
C\left(s^{2}+f^{2}+g^{2}\right) h^{2}+2 r\left(f^{2}+g^{2}\right)=0 .
$$

Sul piano $z=0$, si anmulla pure la tensione normale

Per la [5], e quindi per la [6],

$$
\left.N_{::}=\left(\hat{\imath}+i p \hat{\imath}^{\prime}\right) \eta^{\prime}\right)+2\left(\mu+i p \mu^{\prime}\right) \frac{\partial u}{\partial z} \text {. }
$$

Perciò

$$
\lambda+2 u+i p\left(\lambda^{\prime}+2 u^{\prime}\right)=\frac{\rho \nu^{2}}{h^{*}} \quad ; \quad \lambda+i p \hat{\imath}^{\prime}=\left(u+i p u^{\prime}\right)\left(\frac{h^{*}}{h^{*}}-2\right)
$$

E:

$$
N_{n}=\left(u+i u^{\prime}\right)\left\{\left(\frac{h^{2}}{h^{2}}-2\right), 2,2 \frac{\partial w}{\partial z}\right\}
$$

$$
\frac{\partial w}{\partial z}=-\frac{r^{\dot{z}}}{h^{2}} e^{-\cdots}-s C e^{-\mathrm{sz}} \text {. }
$$

La condizione $N_{3}=0$, corrispondente a $z=0$, tenendo conto della [13], dà

$$
h^{2}-2 h^{2}-2\left(r^{2}+h 2 s C\right)-0,
$$

oppure, essendo per la [11]

$$
\begin{gathered}
r^{2}+h^{2}=f^{2}+g^{\mathrm{n}}, \\
l_{i}{ }^{2} \quad 2\left(1^{2}+z^{\mathrm{n}}\right) \quad 2 h^{\circ} \mathrm{s} C=0 .
\end{gathered}
$$

Eliminando $C$ fra [21] e [22], otteniamo l'equazione per la quale il tempo di vibrazione (periodo) ̀̀ determinato in funzione dellis 
lunghezzal d'onda e delle proprietà del solido. Si ha

$$
\begin{aligned}
& C=-\frac{2 r\left(f^{2}+g^{2}\right)}{h^{2}\left(s^{2}+f^{2}+g^{2}\right)}, \text { e sostiluendo in }\lfloor 22\rfloor, \\
& \left.\left\{h^{2}-2\left(f^{2}+g^{2}\right)\right\} \cdot \int_{s^{2}}+f^{2}+g^{2}\right\}+\operatorname{trs}\left(f^{2}+g^{2}\right)=0,
\end{aligned}
$$

oppure, per la $[17]$ - per la cinale $s^{2}+f^{2}+g^{2}=2\left(f^{2}+g^{2}\right)-k^{2}-$

$$
\left\{2\left(r^{2}+g^{2}\right)-l^{2}\right\}^{2}=\operatorname{lrs}\left(j^{2}+g^{2}\right) \text {. }
$$

Quadrando la [23 e inlroducendo i valori di $r^{2}$ e $s^{-}$dali dalle [11], [17] abbiamo

$$
\left\{2\left(f^{2}+g^{2}\right)-k^{2}\right\}^{t}=16\left(f^{2}+g^{2}\right)^{2}\left(f^{2}+g^{2}-h^{2}\right)\left(f^{\prime \prime}+g^{2}-h^{2}\right) .
$$

Posiamo scrivere anche

$$
h^{2}\left\{1-8 \frac{f^{2}+g^{2}}{h^{2}}+\left(21-16 \frac{h^{2}}{l^{2}}\right) \frac{\left(f^{2}+g^{2}\right)^{2}}{h^{2}}-16\left(1-\frac{h^{2}}{h^{2}}\right) \frac{\left(j^{2}+g^{2}\right)^{3}}{k^{5}}=0 .\right.
$$

Dalle [19], ricordando le [20] e [21], in ha

da cui

$$
u=-\frac{i f}{h^{2}} e^{-r 2}+\left[\frac{2 i f r}{s h^{2}}+\frac{i f}{s h^{2}}\left(-\frac{2 r\left(f^{2}+g^{2}\right)}{s^{2}+f^{2}+g^{2}}\right)\right] e^{--\mu},
$$

$$
h^{2} u=i l\left[-e^{-r m}+\left(\frac{2 r}{s}-\frac{1}{s} \frac{2 r\left(y^{2}+g^{2}\right)}{s^{2}+f^{*}+g^{2}}\right) e^{-x}\right] \text {. }
$$

Tra parenlesi quadra può scriver-i $\frac{2 r}{s}\left(1-\frac{f^{2}+g^{2}}{s^{2}+f^{2}+g^{2}}\right)=\frac{2 r s}{f^{2}+s^{2}+g^{2}}$.

Analogamente per $h^{2} x$. Percio, in wenerale sarà -.. preseindendo ditgli esponenziali -

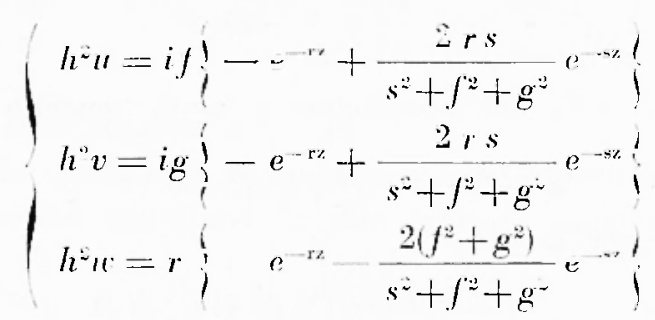

Se consideriamo il moto soltanto in due dimensioni, e precisitmente nel piano principale, posiamo porte $y=0$. La [21] diviene allora 


$$
\left.1-8 \frac{f^{2}}{h^{2}}+\left(24-16 \frac{h^{2}}{h^{9}}\right) \frac{f^{-1}}{h^{4}}-16\left(1-\frac{h}{h^{2}}\right) \frac{f^{j}}{h^{5}}=0 . \quad 2^{\prime}\right]
$$

Per $g=0$, è inoltre $v=0$, e le [24] divengono

$$
\left\{\begin{array}{l}
\left.\left.h^{2} u=i f\right\}-e^{--x}+\frac{2 r s}{s^{2}+f^{2}} e^{-\alpha}\right\} \\
h^{2} u=r \mid
\end{array}\right.
$$

E' ancora. sempre per $g=0$, o pois $\frac{f^{2}}{k^{2}}=y^{-}$(radice della $\left[\underline{2} \underline{1}^{\prime}\right]$ )

$$
\begin{aligned}
& r^{2}=f^{2}-h^{2}=h \dot{\alpha} \gamma^{2}-h^{2}=k^{2}\left(\lambda^{2}-\frac{h^{2}}{h^{2}}\right) \\
& s^{2}=f^{2}-l^{2}=k \sim\left(\chi^{2}-1\right) \\
& 2 r s=2 k^{\sim} \sqrt{\gamma^{2}-\frac{h^{2}}{k^{2}}} \sqrt{\gamma^{2}-1} ; s^{2}+f^{2}=2 f^{2}-k^{2}=h\left(2 \gamma^{2}-1\right) \text {, } \\
& \frac{2 r s}{s^{2}+f^{2}}=\frac{2 \sqrt{x^{2}-\frac{h^{2}}{k^{2}}} \sqrt{\psi^{2}-1}}{2 \%^{2}-1} ; \frac{2 f^{2}}{s^{2}+f^{2}}-\frac{2 \psi^{2}}{2 \psi^{2}-1}
\end{aligned}
$$

Le $\left[25^{\prime}\right]$ si possono scrivere. introducendo grli esponenziali finora sollintesi,

$$
\begin{aligned}
& \left\{\frac{h^{2}}{h} u=i \% e^{-r 2}+\frac{\left.2 \sqrt{\gamma^{2}-\frac{h^{2}}{h^{2}}}\right|^{h-1}}{2 \psi^{2}-1} e^{\ldots}\right)
\end{aligned}
$$

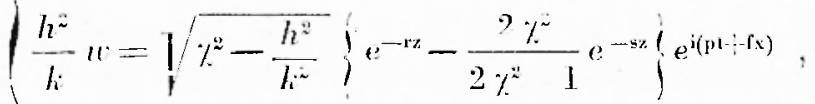

\section{opptire}

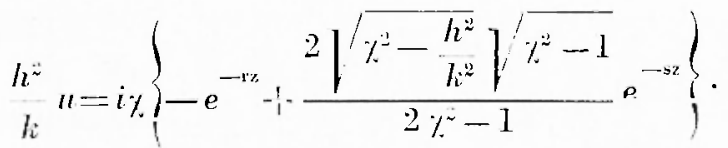

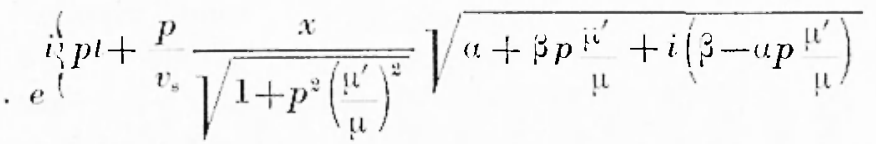

$$
\begin{aligned}
& h^{2} w=\sqrt{\left.\left.h^{2}-\frac{h^{2}}{k^{2}}\right)^{-\mathrm{rz}}-\frac{2 \psi^{2}}{2 \gamma^{2}-1} 0^{-\infty}\right\}} \text {. }
\end{aligned}
$$

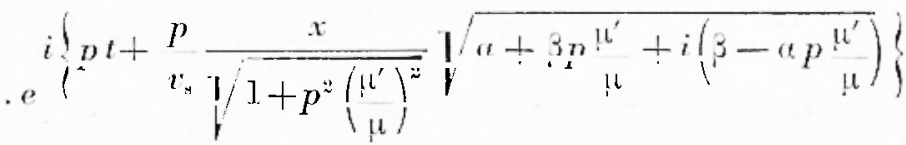


dove u, $\beta$ sono la parte reale e il coefficiente dell’imaginatio delle -oluzioni della [24'], corrispondenti alle onde di Rayleigh e $v$ rap. presenta la velocila delle onde trasversali nel mezzo consideralo.

2. Pariamo ora ai valori numerici.

Risolviamo a duesto scopo la $\left[24^{\prime}\right]$, yicordando le espressioni di $h^{2}$ a $l^{2}$ a noll'ipotesi di Poisson. Si sil rhe $h^{\prime}=-\frac{2}{3} u^{\prime}$. Poslo

lat $\left\lceil 24^{\prime \prime}\right.$ diviene

$$
\frac{l_{i 2}^{2}}{f^{2}}=\varepsilon
$$

dove

$$
\varepsilon^{\prime \prime}-8 \varepsilon^{2}+(24-16 \varphi) \varepsilon-16(1-\varphi)=0,
$$

$$
\varphi=\frac{h^{2}}{k^{2}}=3 \frac{9\left(\frac{\mu}{\mu^{\prime}}\right)^{2}+4 p^{2}}{81\left(\frac{!^{\prime}}{\mu^{\prime}}\right)^{2}+16 p^{2}}+i \frac{15 p \frac{\mu^{\prime}}{\mu^{\prime}}}{81\left(\frac{\mu}{\mu^{\prime}}\right)^{2}+16 p^{2}},
$$

f ron le nlecriori posizioni:

$$
r=y+\frac{8}{3} \quad, \quad y=1-\frac{j}{16},
$$

la $[2-1 . "]$ i trasforma nell'equazione

$$
{ }^{3}{ }^{3}+\left(j-\frac{40}{3}\right) \psi+\left(\frac{5}{3} j-\frac{448}{27}\right)=0 . \quad\left[24^{\prime \prime}\right]
$$

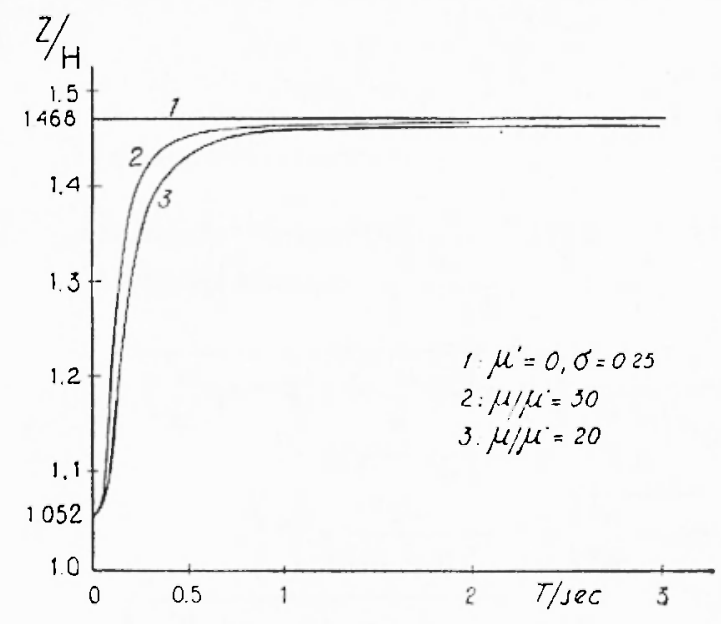

Fir. 1

Adidamento del rapporto $Z / H$ prescindendo dall aś-orbintento: andamento analogo. fra gli stessi estremi. nel raso in cui si consideri l'assorbimento
La lisoluzione della [2t'"'] è slala proposta al bencmerito Istituto Italiano per le Applicarioni del Calcolo. I risultali dei raicoli per vari valori del rapporto $n / \mathrm{u}^{\prime}$ di $T$, sono riportati nelle tabelle I, II e III. In csie $\gamma$ e $\delta$ sono la par. le reale e il coefficiente dell'imateinario del rapporto $\frac{h^{2}}{h^{2}} ; k \psi_{1}, t \%_{1}$, $R \%_{2}, I \%_{2}, \quad$ " e $\beta$ la parte reale e il coefficiente dellimaginario 


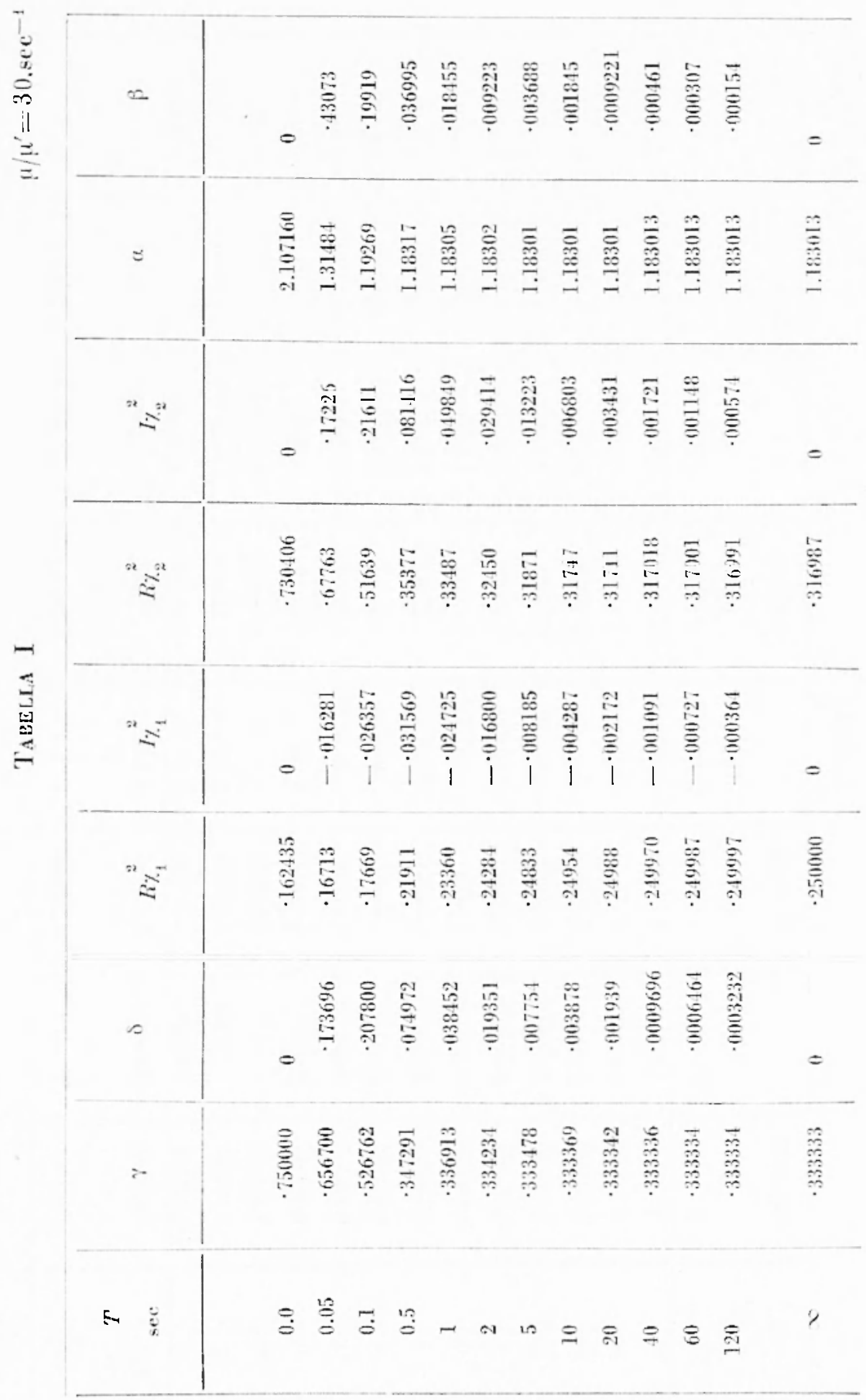




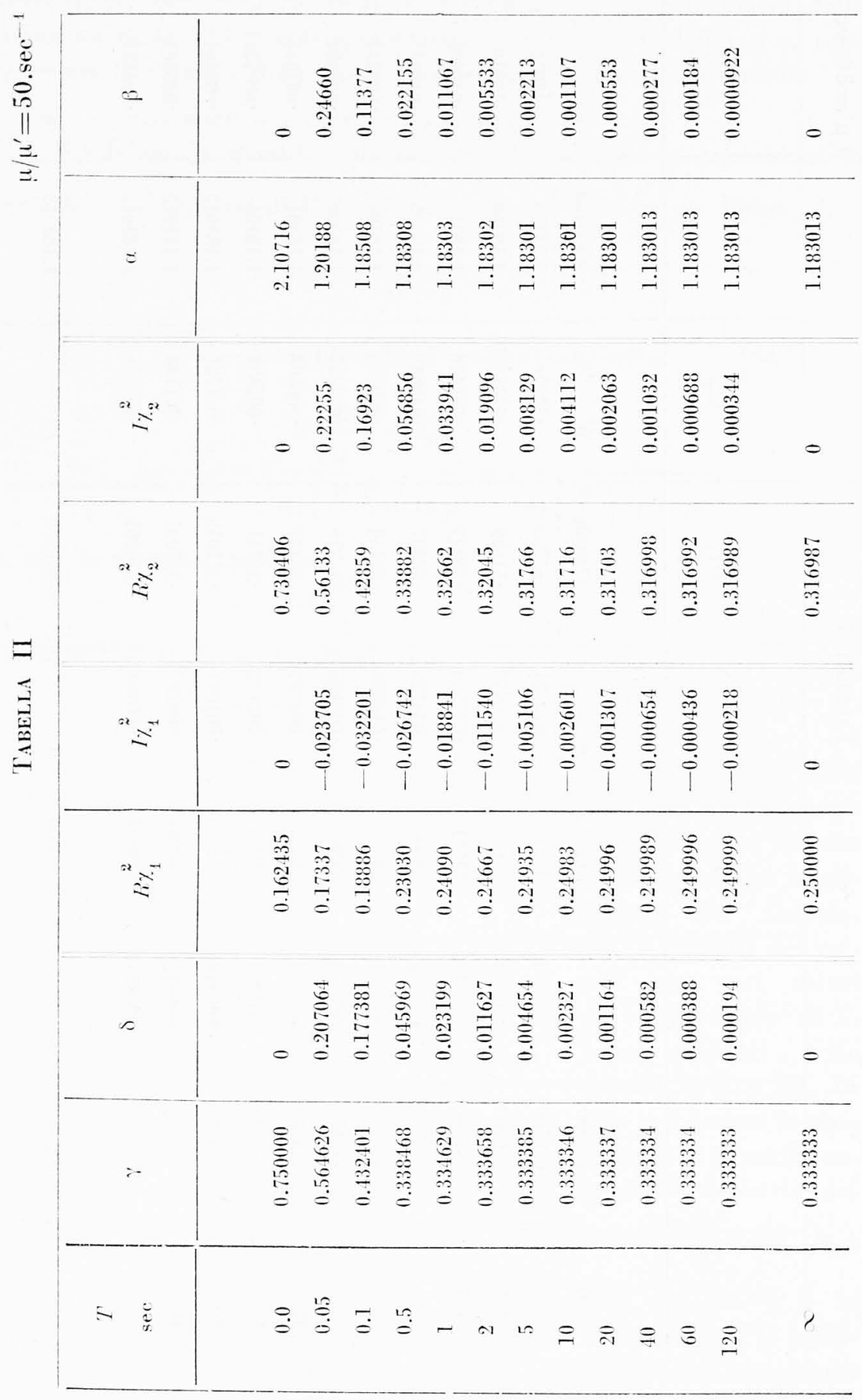




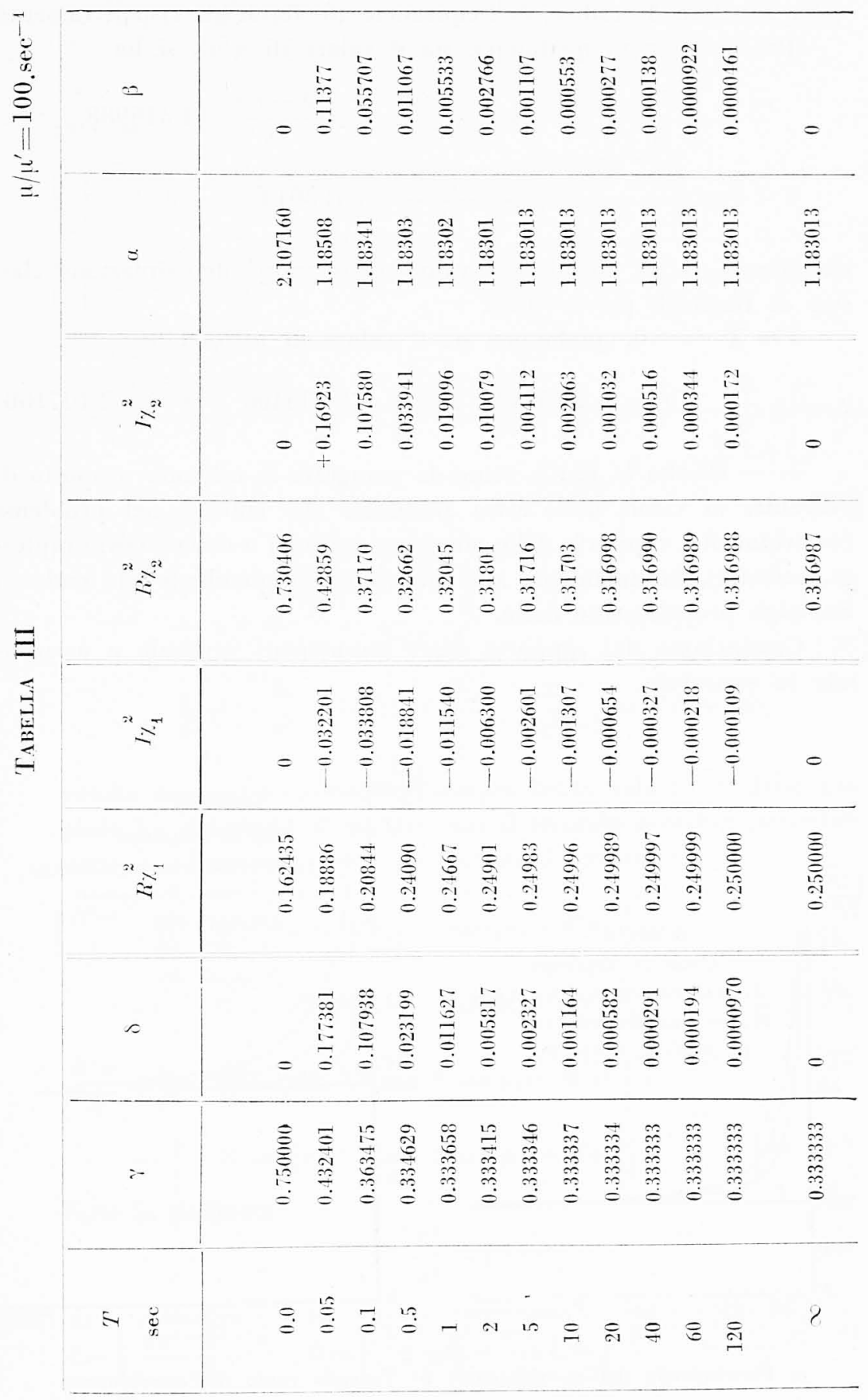


della $1^{2}$, 2" e $3^{\text {it }}$ radice dell'equazione di Rayleigh rispellivamente. Per $T \rightarrow \infty$. qualunque sia il valore di $u / \iota^{\prime}$, si ha

$$
\begin{gathered}
\gamma-\frac{1}{3}, \quad z_{1}^{2}=\frac{1}{4}, \frac{3-13}{4}=1,316988, \\
4-\frac{3+\sqrt{3}}{4}=1,183013
\end{gathered}
$$

che sono - i tre ultimi - i ralori delle radici dell'eruazione ela:sieal di Rayleigh per $\sigma=0,25$.

Per $T \rightarrow->0$, qualumque sia il valore di $! 1 / \iota^{\prime}, \therefore$ ha

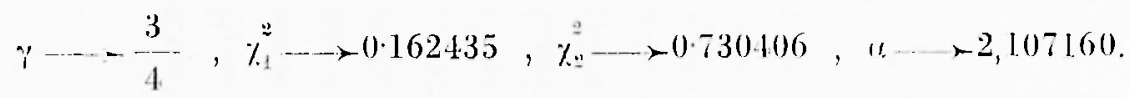

3. - Risolta la $\left[24^{\prime}\right]$, come da patagrafo 2. mi sono proposto di pervenire ai valori delle altre grandezze the entrano nel problema (assorbimento, rapporto delle ampiezze verticali e orizzontali, ampiezzal, velocita), limitatamente alle soluzioni rorrispondenti alle onde di Rayleiggh propriamente delte.

Cominciamo dal rapporlo degli postamenti verticale e orizzontale in superficic.
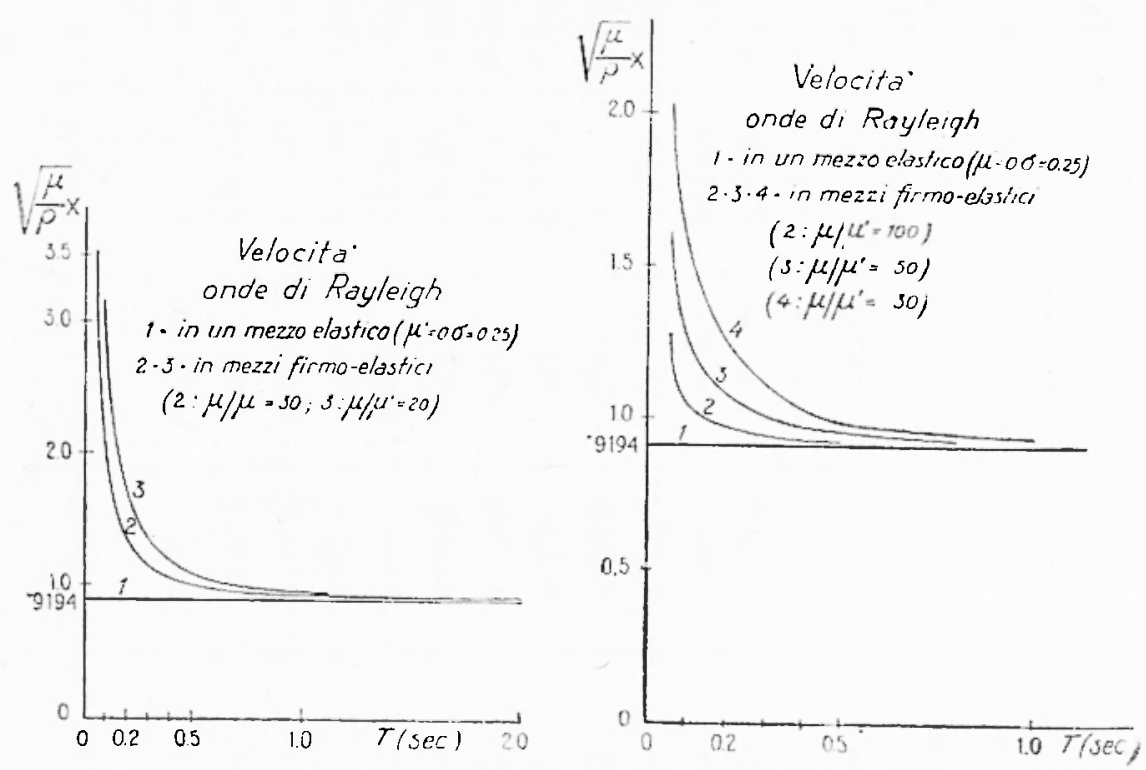

Fig. 2

a) Prescindendo dall'acorbimento; b) Tenemdo conto dell'asorbinento 
Po-10, nell eeponenziale dellat $\left[25^{-1 \prime \prime}\right]$

avremo

$$
a=a+\beta p \cdot \frac{\mu^{\prime}}{!} \quad, \quad b=\beta-u p \frac{\mu^{\prime}}{! !},
$$

$\left\{\begin{array}{l}\sqrt{a+i b}=\left(\sqrt{\frac{\sqrt{a^{2}+b^{2}}+a}{2}}+i \sqrt{\frac{\sqrt{a^{2}+b^{2}}-a}{2}}\right) \text { se } b>0 \\ \sqrt{a+i b}=\left(\sqrt{\frac{\sqrt{a^{2}+b^{2}}+a}{2}}-i \sqrt{\frac{\sqrt{a^{2}+b^{2}}-a}{2}}\right) \text { se } b<0 .\end{array}\right.$

Sia ancora

$U=\sqrt{\frac{\sqrt{a^{2}+b^{2}}+a}{2}}, \quad V=\sqrt{\frac{\sqrt{a^{2}+b^{\prime \prime}}-a}{2}}, \|=-\frac{l}{r \sqrt{1+\left(p \cdot !^{\prime \prime}\right.}}$

e inoltre

$$
\begin{aligned}
& -\%+2 \frac{\% \sqrt{\psi^{2}-\frac{h^{2}}{h^{\prime \prime}}} \sqrt{\varkappa^{2}-1}}{2 \%^{2}-1}=-R+i s \\
& \sqrt{\varkappa^{2}-\frac{h^{2}}{h^{2}}}-\sqrt{\%^{2}-\frac{h^{2}}{h^{2}}} \cdot \frac{2 \%^{2}}{2 \%^{2}-1}=-R+i s^{\prime},
\end{aligned}
$$

poiché nei cati considerati is empre $b<0$ ), vale la 2" delle [20] lia quale ha significalo fi-ieo solo con il secondo membro precedulo disl segno - Pertanto, per $z=0$, possiamo scrivere

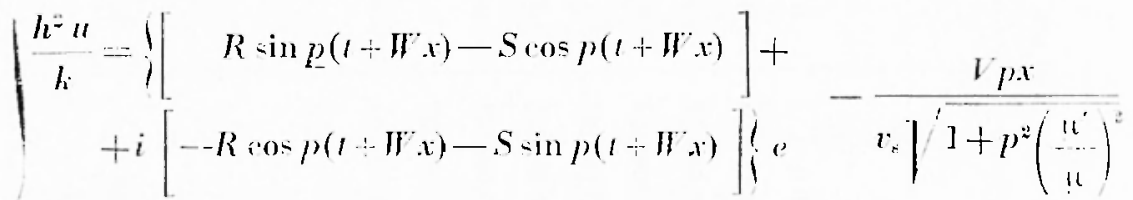

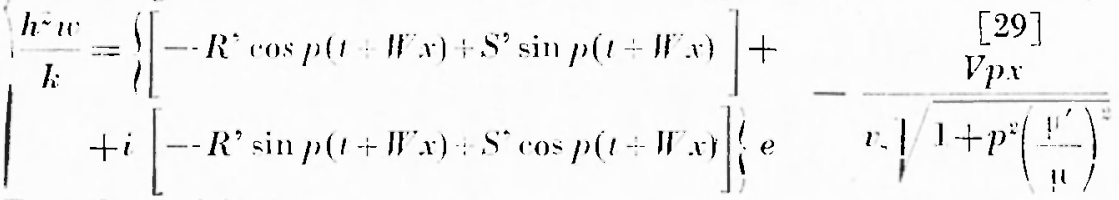

Fitie le posizioni

$$
\frac{h^{2}}{h^{2}}=i+i \delta:
$$

$$
A=\sqrt{\frac{\sqrt{u^{2}+\beta^{2}+a}}{2}}, B=\sqrt{\frac{\sqrt{a^{2}-\beta^{2}-a}}{2}} ; C=\sqrt{\frac{\sqrt{(u-\gamma)^{2}+(\delta)^{2}}(u-)^{2}}{2}} \text {. }
$$




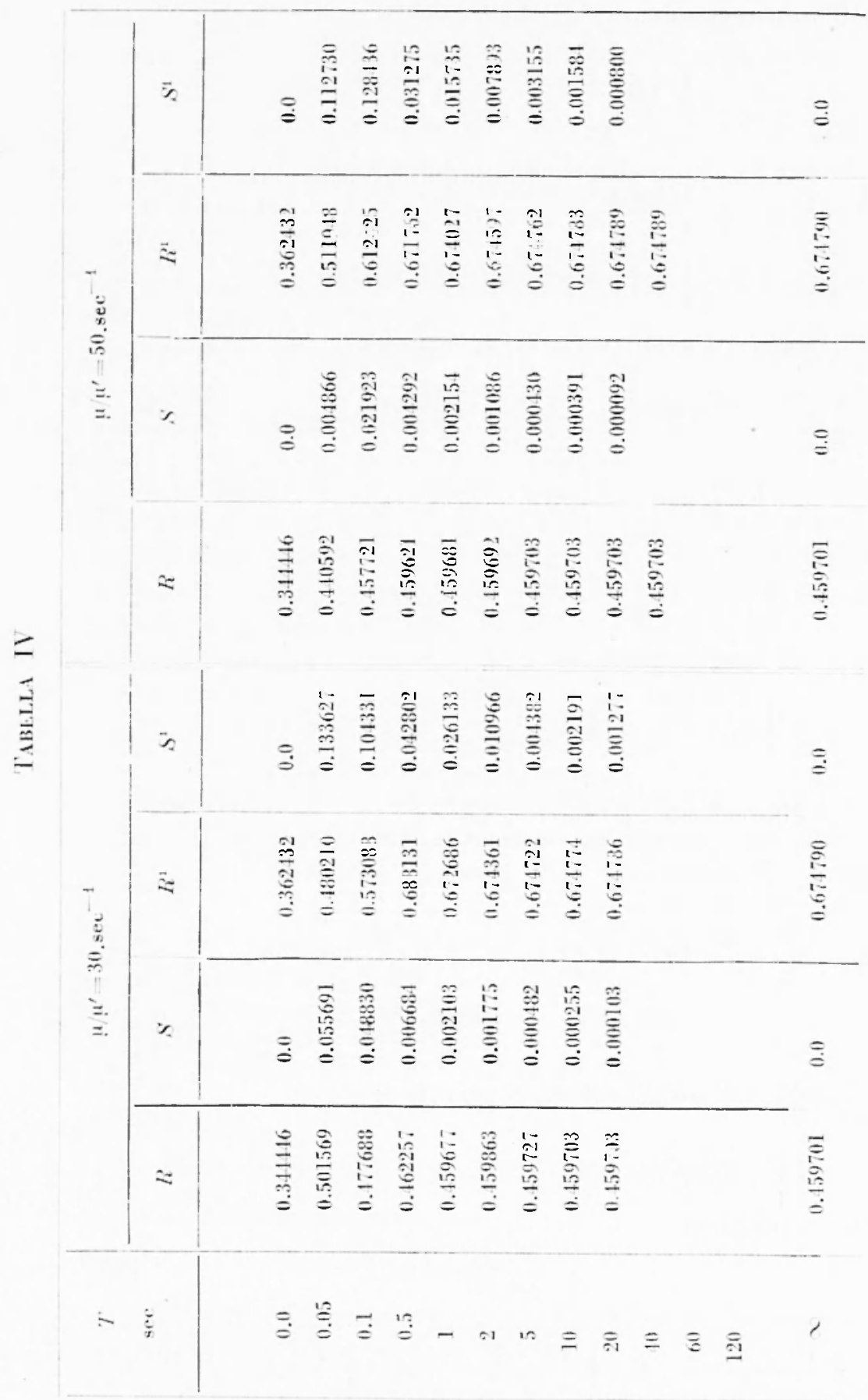




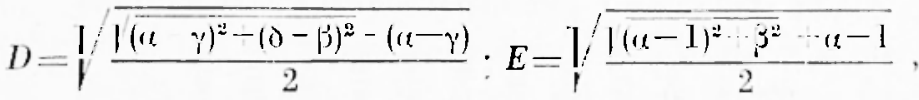

$$
\begin{aligned}
& F=\sqrt{\frac{\sqrt{\left(\overline{(1)^{2}+\beta^{2}}-\alpha+1\right.}}{?}} ; g=C E+D F \quad, \quad h=C F-D E \text { : }
\end{aligned}
$$

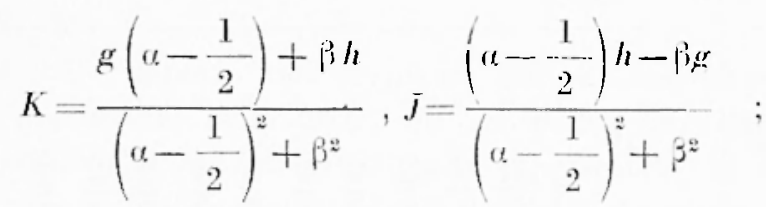

$$
\begin{aligned}
& L=-\frac{\alpha\left(\alpha-\frac{1}{2}\right)+\beta^{2}}{\left(\alpha-\frac{1}{2}\right)^{2}+\beta^{2}}, M=-\frac{0,5 \beta}{\left(\alpha-\frac{1}{2}\right)^{2}+\beta^{2}} \\
& N=A K-B J, O=B K+A J ; P=C L+D M, Q=C M-D L,
\end{aligned}
$$
consegue $($ per $\beta<\delta)$

$$
\begin{aligned}
& \%=A+i B, \sqrt{\%-\frac{h^{i}}{l^{*}}}=C-i D, \sqrt{\varkappa^{2}-1}=E+i F, \\
& \sqrt{\%^{*}-\frac{h^{2}}{h^{2}}} \sqrt{\psi^{2}-1}=g+i h ; 2 \frac{\sqrt{\varkappa^{2}-\frac{h^{2}}{h^{2}} \cdot \sqrt{\%^{2}-1}}}{2 \%^{2}-1}=\Lambda+i J ;
\end{aligned}
$$

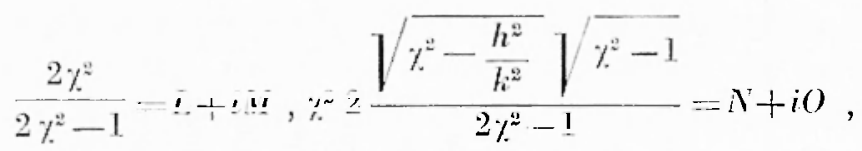

$$
\begin{aligned}
& \sqrt{\varkappa^{2}-\frac{h^{2}}{h^{2}}} \cdot \frac{2 \chi^{2}}{2 \chi^{2}-1}=P+i Q \cdot\left(^{*}\right)
\end{aligned}
$$

Pertanto

$$
R-A-N, S=O-B ; R^{\prime}=P-C^{\prime}, S^{\prime}=-(Q+D) .
$$

Owiamente, la parte reale delle [29] is data da

$$
\begin{aligned}
& \frac{h^{*} u}{k}=\{R \sin p(t+W x)-S \cos p(t+W x)\} e^{--\frac{V p x}{v_{0} / \sqrt{1+p^{2}\left(\frac{l^{\prime}}{!}\right)^{2}}}} \\
& \frac{h^{2} u}{l}=\left\{-R^{\prime} \cos p(t+\pi x)+S^{\prime} \sin p(1+W x)\right\} e-\frac{r_{p x}}{r_{r} \sqrt{1+p^{2}\left(\frac{\mu^{\prime}}{\mu}\right)^{2}}}
\end{aligned}
$$

(*) Per $\beta>0$ i $\sqrt{\gamma^{2}-\frac{h^{2}}{h^{2}}}=\mathrm{C}+\mathrm{i} D, g=\mathrm{CL}-\mathrm{DF}, \mathrm{h}=\mathrm{CF}+\mathrm{DE}, \mathrm{P}=\mathrm{CL}-\mathrm{DM}$, $\mathrm{Q}=\mathrm{DL}+\mathrm{CM}$. 


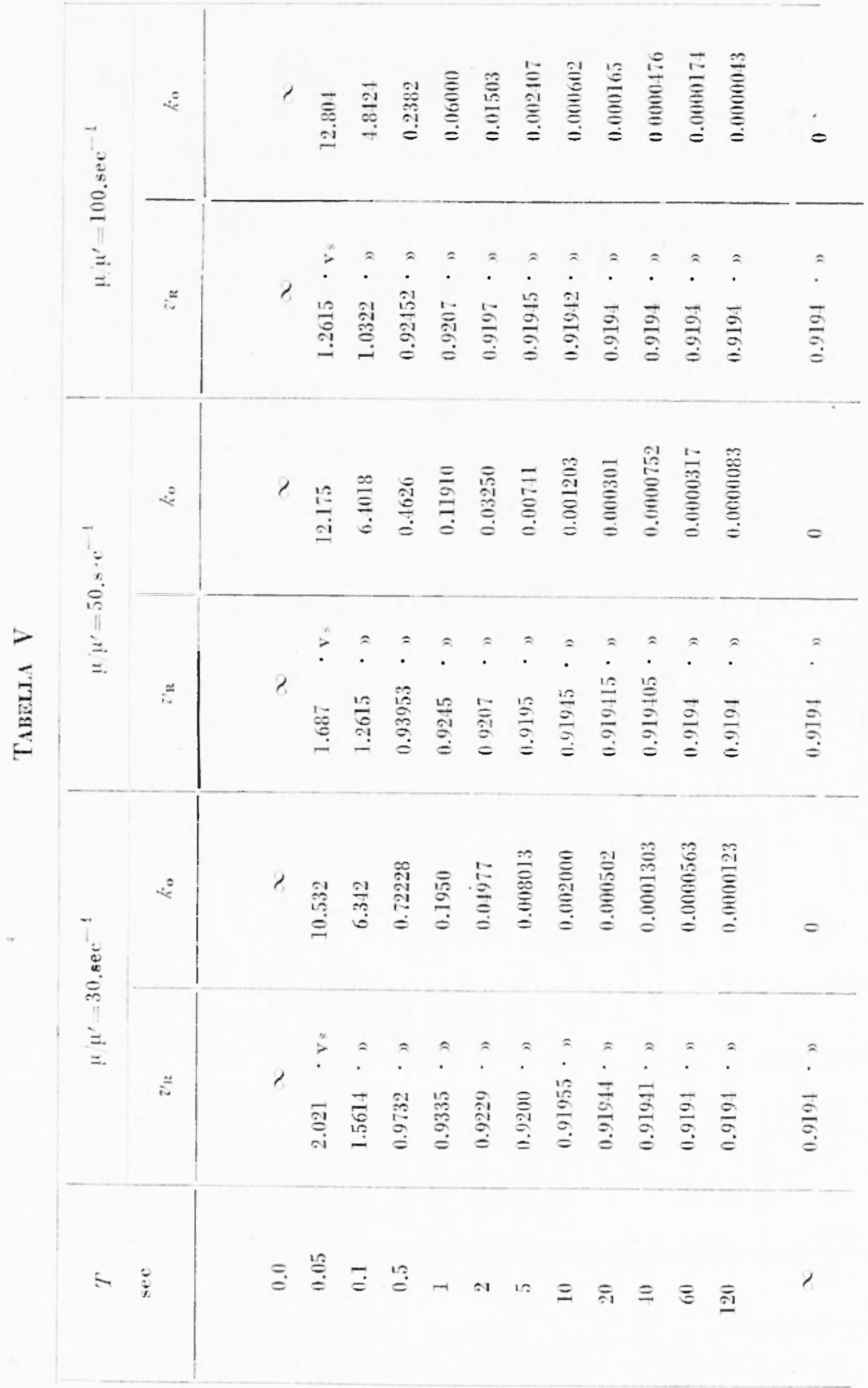


Nella Tabella IV sono contenuti i valori $R, R$, S, S', per vari valori di $\mathrm{T}$, in corrispondenza di $u / \iota^{\prime}=30$ e $! / \iota^{\prime}=50$. Dit esail risulta che, esclusi i periodi più piccoli (di poco interese per le onde di Rayleigh propriamente detie), $S$ e $S^{\prime}$ assumono valori via via decresenti, tendent allo zero: trascurabili comunque nei confronti di ruelli di $R, R^{*}$,

Pertanto. il rapporlo dell ampiezza del movimento verlicale a quella del movimento orizzoniale, da un valore prossimo all'unità 1,052 per periodi pressoché nulli, tende rapidamente al valore 1.47 rhe le compete nella classica tcoria di Rayleigh in mezzi puramente elastici; tanto pià rapidamente, quanto maggiore è il rapporto u/u'.

1. - Passiamo alla velocità. Essa vale evidentemente

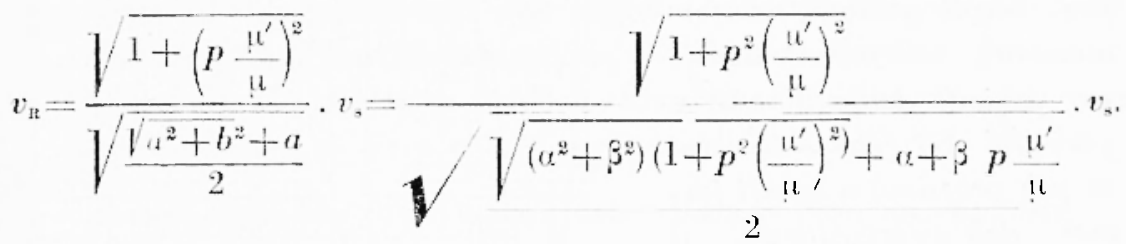

La Tabella $V$ contiene $i$ risultati dei calroli per i valori 30 , 50 e 100 del rapporto $1 / \iota^{\prime}$, al variare del periodo. Si nola subito the, da un valore teoricamente infinito per $\mathrm{T}=0$, la velocità delle onde di Rayleigh in un mezzo firmo-clastico tende al valore che essa assume in un mezzo puramente clastico col tendere del periodo allinfinito, e tanto più rapidamente quanto maggiore ̀ il valore del apporto $11 / 41$.

Questo risuliato era già stalo ottenuto nel lavoro precedente; reta quindi confermato una sorta di effelto della firmo-viscosita sulla propargazione delle onde superficiali, che appare come propagazione inomala.

5. - Veniamo infine all'assorbimento di un mezzo furmo-elasico. Il coefficiente di assorbimento è

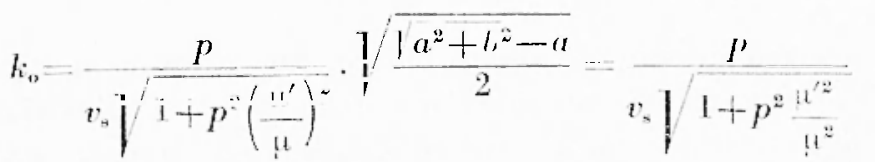

$$
\begin{aligned}
& \sqrt{\sqrt{\left(\alpha^{2}+\beta^{2}\right)\left(1+p^{2} \frac{u^{\prime 2}}{u^{2}}\right)}-\alpha-j p \frac{u^{\prime}}{u}} .
\end{aligned}
$$


Falto $\mathrm{s},=3.3 \mathrm{~km} / \mathrm{ser}$. velorita modia delle onde ma-versali nello strato -uperfiriale lerrestre, o asecegnati al !t/!!' i valori 30, 30, 100 ripeltivamente i calcoli hanno fornito, per periodi diversi, i valori riportali nellal 'Taloclla V.

1 periodi nulli corrisponde quindi un assorbimento infinito, il quale resua comunque elezatissimo per piecoli periodi, conformemente all'osservazione: per periodi dellordine $20^{\mathrm{s}} \mathrm{s}$ p'r $1 \mathrm{u} / \mathrm{u}^{\prime}=50$ (che is il valore più prossimo a quelli forniti dall'osservazione), il coefficicnte di assorbimento diviene dell'ordine di 0.0003 .3$. che is appunto quello generalmente osseriato.

L'aturito incerno miustifrea quindi pienamente le malatterifiche osservate per Ir onde superficiali, le quali nella teoria di Rayleigh

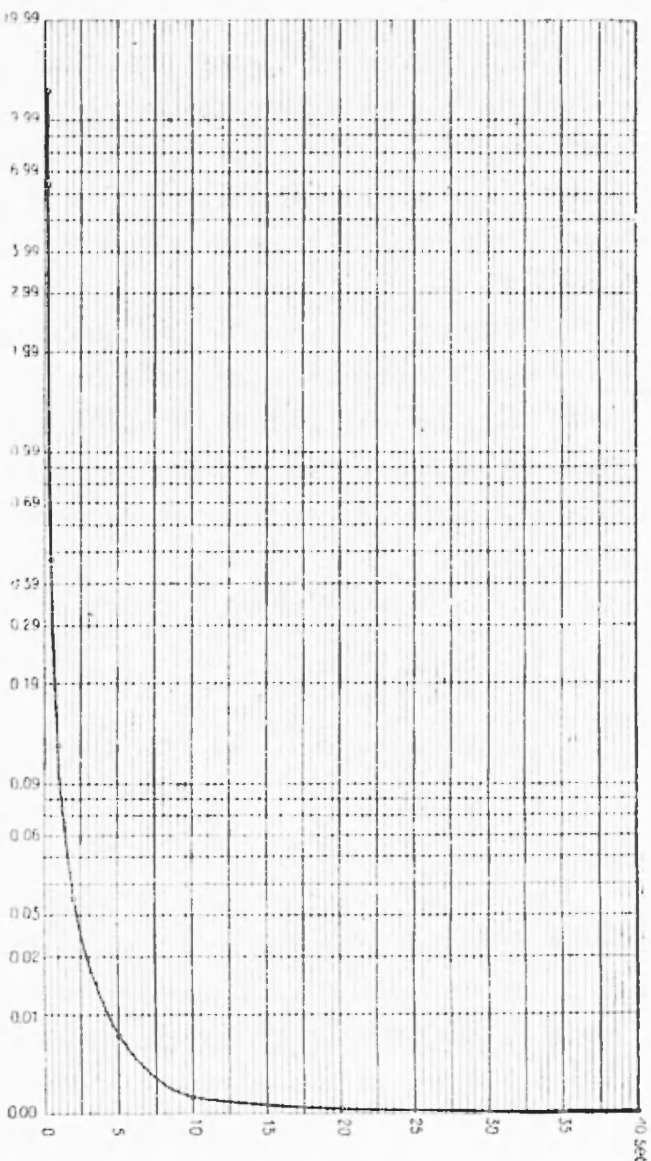

Fig. 3

Andianento del fattore dissorlimento lio nel *iso $! 4 ! !^{\prime}=50$ (Tab). V)

erano considerate come persistenti. Ritengo sia questo uno dei ri-ultati piì notevoli della ricerea.

Roma - Istituto Nazionale di Geofisica -- Luglio 1918.

\section{RI ISSUNTO}

La ricerea oggello di questo laworo, ha condolito ai seguenui risultati: la velociti di propagazione delle onde di Rayleigh in un mezzo firmo-clastico, da un valore infmito per periodi aulli. tende rapidamente, per periodi crescenti, al valore che le compete in mes:i puramente clastici, tamto più rapidamente quamıo maggiore ì il talore del rapporto "l/ $\mathrm{I}^{\prime}$ lı costante di Lamé. $\mathrm{u}^{\prime}$ coefficiente d'allrito 
interno equicoluminale); la firmo-clasticità dotermina una sensibito riduzione della componente verticale del moto. riduzione che i tanto maggiore quanto più piceoli sono il rapporto $\frac{\mathrm{ll}}{\mathrm{u}^{\prime}}$ e il periodo propria dellonda: per onde originanti con grossi periodi lazione de!liattrito interno sul rapporto delle ampiczas e pressocché nulla e il mezao si comporta come puramentc clastico. La firmo-clasticità mò tariare il rapporto delle ampiezze $\mathrm{Z} / \mathrm{H}$ da un valore prossimo all umilà $(1,05)$ a 1,41 , valore che la teoria Rayleigh-Lamb assegna a quel rap)porto per $\sigma-10.25$.

La ricerca ha consentito inoltre di chiarire teoricamente un altro aspetlo delle onde superficiali finora rimasto insoluto. Nella classica leoria di Rayleigh, le onde che portano il suo nome sono considerate come libere, persistenti, non soggdtle ad assorbimento da parte del mezzo. Ora. l'osservazione prova che lassorbimcnto e fortissimo pe: i periodi più piecoli e tende a decrescere rapidamente per raggiungere il valore di circa $e^{-0,0003}$ per periodi dellordine di $20^{\circ}$. La muora coria prova che in un mezzo firmo-clastico (con un rapporto $\left.\frac{\mu}{u^{\prime}}=5^{i}\right)$ sec: ${ }^{-1}$. conforme alla media dei valori osservali) cio is pienamertio dimostrato. Ed e simtomatico che le fondamentali caratleristiche delle onde di Rayleigh trovino così completa giuslificazione in un mezzo indefinito non stratificato.

\section{BIBLIOGIRAFIA}

(1) Ravisigu Loks: On nates propagaled along the plane surface of an elastic solit - Scientific Papers. 11. pagg. 4t11-117.

(2) LAnu H.: On the propagation of tremors ocer the surface of an elastic solid - Philosophical Transactions, CCIII. A. 1901.

(ii) Calor P.: Sulle onde di Rayleigh in un mezzo elastico, firmo-riscoso indefinilo - Pontificia Academia Scientiarum, Acta, X, 1946. 